\title{
Pediatric Intensive Care Unit rounding improvements
}

\author{
Kirsten Beck, Elaine Albert, Andrew Johnsen, Courtney Newhouse, John McGuire
}

To cite: Beck K, Albert E, Johnsen A, et al. Pediatric Intensive Care Unit rounding improvements. BMJ Quality Improvement Reports 2016;5:u208902.w3600. doi:10.1136/bmjquality. u208902.w3600

Received 26 May 2016 Revised 7 June 2016

\section{CrossMark}

Seattle Children's Hospital, USA

\footnotetext{
Correspondence to Kirsten Beck kirsten.beck@seattlechildrens. org
}

\begin{abstract}
Medical rounds in the Pediatric Intensive Care Unit (PICU) took about four hours each day to complete. The length of rounds was affecting the advancement of care of the patients, the engagement of sub-specialty providers who needed to be present on other rounds, and the engagement of the PICU faculty and staff due to overburdening waste created by the long duration of rounds.

Specific interventions were identified aimed at reducing the duration of rounds each day, increasing engagement of the rounding team and satisfying the needs of the patients and families. Post-improvement results were that rounding times were reduced to two hours each day and were pre-scheduled for families, the burden of excess work was lifted for attending physicians, and the presentation expectations during rounds were specified by role. Quality and safety were improved through standard work and auditing.
\end{abstract}

\section{PROBLEM}

The PICU, located at Seattle Children's Hospital, Seattle, WA, USA, has 32 beds and admits approximately 2000 patients each year. Care is provided for medical and surgical patients with a wide range of disease processes. The unit is staffed by 23 board certified Pediatric Critical Care Medicine attending faculty, 10 Pediatric Critical Care Medicine Fellows, and a nursing staff of 300. Additionally, each month six-eight pediatric residents rotate through the PICU. Other members of the care team include unit-based Pharmacists, Nutritionists, Social Workers, and Respiratory Therapists. Daily patient rounds are conducted each morning to review and evaluate patient data from the night before and develop care plans for the day.

The problem statement was that rounds were too long, variable in content and did not reliably advance the daily plan of care. There was variation in length and content between PICU attending physicians and unclear expectations for the purpose of each person's role in rounds.

Rounds in the PICU took nearly four hours each day to complete and included at least ten different roles on the care team. Twice in the last five years, various PICU physicians had made attempts to improve rounds without sustained results. The inability to sustain improvements was attributed to the lack of formal quality improvement training for members of the care team, lack of agreement about the purpose of rounds, missing focus on patient and family needs, limited leadership support, and the absence of data to assess the impact of changes.

\section{BACKGROUND}

In preparation for a comprehensive and structured quality improvement project, a literature search was performed looking for best practice ideas and only one reference was found (see ref. ${ }^{1}$ ). Direct observations of daily rounds were completed, conversations occurred with colleagues in other medical units who had made recent changes, and interviews were conducted with stakeholder groups. Additionally, data was obtained via an online survey of the care team $(\sim 140$ respondents) about their understanding of the purpose, length, content of, and expectations for their participation in rounds.

The project was initiated by a multidisciplinary PICU rounding improvement team of physician and nursing leaders, a lean consultant, and a project manager. Implementation was carried out by the PICU care teams including attending physicians, fellows, residents, nurses, pharmacists, and nutritionists (See supplementary file - Process Map).

\section{BASELINE MEASUREMENT}

The PICU rounding improvement team chose simple metrics that could be measured and that correlated to purpose and goals. Baseline data was established through direct observation and the summary is as follows. The ultimate goal was to decrease the length of rounds from four hours to two hours within six months of initiating the formal QI project. The team used self-audit rounding time data for measurement. 
A written Plan of Care was not present for each patient such that nurses could plan for delivering care and families would know goals of care for their child in the PICU that day. Use of this written document was implemented near the beginning of the QI project as it was a key part of the project aim.

For several years, there has been an expectation throughout the Seattle Children's Hospital that daily rounds will include a review of patient care Quality and Safety indicators relevant to the individual unit. For the PICU, these include removal of catheters and tubes that are no longer needed, ordering of interpreters for non-English speaking families and discontinuation of unnecessary lab and other diagnostic tests. Before the project began, there was no documentation for ensuring that all of the Quality and Safety questions were asked for every patient every day and thus it was decided to incorporate this best practice into rounds.

Anecdotally, it was thought that patient orders were read back to the team for clarity $100 \%$ of the time but this had never been documented. By making order read back part of the Quality and Safety checklist, it ensured that this important patient safety practice occurred and was able to be audited. The hospital uses a computerized physician order entry system.

For various reasons, patient/family satisfaction scores were on the decline and were not meeting the goal. Seattle Children's uses a series of questions that were developed by the National Research Corporation and are asked via a phone or email survey after discharge.

Many of the rounding team members were unclear about what information they should be relaying during rounds and this led to duplication and waste in the process and a high level of variability from week to week.

As a balancing measure, the team wanted to ensure that relevant education continued for trainees and so the team monitored available Resident Teaching Score data collected by the Graduate Medical Education office.

In summary, we had qualitative dissatisfaction with rounds, but only limited quantitative data to form the basis for measurable improvement.

\section{DESIGN}

The team's clinical leaders set the stage for improvement by stating three goals: 1) the purpose of rounds is to advance the care of the patient, 2) to efficiently use the time of all the people on the care team, and 3) reduce rounding time from four hours to two hours. This time target was selected based on observations that some members of the PICU faculty were routinely able to complete rounds in as little as two hours, whereas the majority of the faculty had rounds lasting three-four hours or more. The project leaders created a sense of urgency by describing the current state, presenting relevant rounding data to care team members, managing change by being part of the solution and leading by example. Many interventions were trialed and reasons for success and failure were documented. Leaders and early adopters alike trialed improvement ideas then rolled them out to the rest of the team once deemed successful.

Two specific tools were created 1) a standard Plan of the Day (POTD) template (see Strategy section) which includes a checklist for review of Quality and Safety indicators and 2) a formal rounding schedule which provided families with the specific time when the care team would be arriving at the room to discuss and plan their child's PICU care. In addition to establishing a schedule, this tool served many purposes. It provided an opportunity for self-auditing, afforded longitudinal data collection, and allowed subspecialty providers and bedside nurses to know when their patients were scheduled for rounds, thus decreasing time spent waiting for care team members to join rounds. Faculty agreed to trial the process for one month. While it represented additional work for attending physicians, the benefits to nursing staff and families and the ability to recognize when rounds were being delayed outweighed the extra time and effort to complete the schedule each morning. Hence, faculty agreed to adopt it as standard work.

Other interventions included collecting and displaying data around duration of rounds, posting the plan of the day in the patient room, ensuring that order read-backs (accuracy of each order verbally confirmed) happen for every patient and, lastly, creating standard work (scripting) for what specific information is to be presented on rounds role (e.g. bedside nurse, pharmacist, nutritionist, resident, etc.). Additionally, complex family conversations and the majority of academic teaching were to be taken "off-line" or "outside of rounds". This practice allowed for increased focus on each of these later in the day, while keeping morning rounds focused on making and implementing the plan of care.

Soon, a culture of improvement developed, changes occurred, and suggestions for improvement were coming in from nurses, physicians, and other members of the PICU team as engagement grew.

\section{STRATEGY}

Several pieces of standard work were created for the care team to be successful.

\section{RESOURCE ATTENDING ROLE:}

A new role was created to off-load some of the work from the two primary care teams, especially during rounds.

PDSA cycle 1: The aim was to better use an additional attending role to advance patient care. In the prior state, the additional attending was on "standby" in a backup role and waited in the PICU to be called. In reality, the backup attending was rarely called for routine work and the rounding teams were frequently interrupted, thus adding to the time of rounds. The redefined "Resource attending" role was piloted by the clinical physician leaders of the implementation team. 
The expectation was set for the Resource attending to assist the primary care teams with unit management (i.e. sedation, procedures) and manage critically ill patients outside the PICU (i.e. code blue, emergency department consultations, transport calls) and manage admission of new patients to the unit during rounding times. The hypothesis was that by switching from a "push" model of delivering work to the Resource attending to a "pull" model of the Resource attending seeking specific work, the rounding teams would no longer be interrupted and could focus on rounding efficiently. The intervention was a success, and thus, standard work was created and disseminated in the next cycle.

PDSA cycle 2: Standard work defining the responsibilities of the Resource attending role and a written document were created and used to train new faculty in the new role. The hypothesis was that implementing standard work would reduce variation between providers in carrying out their roles and improve overall efficiency of the entire rounding process. We found the Resource attending role to be a predictable and stable part of PICU operations and the role is reported by physicians to be satisfying.

Plan of the Day Form (POTD):

PDSA cycle 1: One improvement team leader created a Plan of the Day (POTD) template which included the agreed upon plan of care, resident contact information, and the Quality \& Safety checklist. The initial aim was for the form to be posted in the patient room. Compliance with completion of the Quality and Safety checklist met the target of $82 \%$ of the time. Beside completion, the team's second goal was to have $90 \%$ of POTDs posted and visible to families and care team members. Compliance was audited for several months, during which the team never met its intended $90 \%$ POTD posting target. Nursing identified an important barrier to posting the POTD; they needed to have the resident contact information readily available to them. Nurses couldn't quickly retrieve the POTD if it was posted in the room; this was particularly relevant for patients under an isolation protocol.

PDSA cycle 2: A new hypothesis was that if the contact information was removed it would improve posting of the POTD forms in the room. However, that information proved to be the most valuable part of the form for PICU nurses. The compromise was to have the POTD available outside the room and thus there is no longer the need to audit. Another unanticipated benefit of the POTD from was that it created accountability around the advancement of patient care. Overnight nurses, who were not involved in daily morning rounds, could review the POTD and prompt the care team to address items that remained incomplete.

PDSA cycle 3: Recently, through changes to the electronic medical record, the Quality \& Safety checklist and contact information are now completed electronically which eliminates the need for a paper form. As a result, the Plan of the Day paper form has been phased out and the team is revising documentation of the daily care plan. Having the checklist in the clinical information system also enables automated auditing of compliance and links completion to a visual alert on the PICU quality and safety dashboard.

Other small interventions such as several revisions to the rounding schedule template, resident and pharmacist rounding scripts, real-time adjustment to subspecialty rounding times, and refinement of Quality and Safety checklist elements were managed through a just-in-time process.

\section{RESULTS}

The team feels that the work yielded significant results that improved the focus of rounds, and thus, the quality of care in the PICU. Rounding times have shortened to our stated goal of two hours and have been relatively stable over the last year. Current variability is deemed appropriate as it reflects the average time given to each patient for acuity and ICU census, which have both increased this year. The average rounding time is 11-14 minutes per patient with the ability to catch up on some patients when care discussions are longer on others. Importantly, we have made the primary sources of daily variability driven by patient complexity, not individual provider preference.

The Plan of the Day (POTD) is filled out for each patient as care decisions are made and is available for the team to review throughout the day.

The addition of the Quality \& Safety questions, in the form of a checklist, is now a routine part of rounds as those questions are reliably discussed on every patient. When audits were collected on a paper form, the checklist completion rate was $82 \%$. Transitioning to an electronic form recently, that better fits the provider online workflow, has improved the completion rate to $100 \%$ and represents a significant improvement from the year prior. Order read-back is driven by the Quality \& Safety checklist and happens reliably on every patient. We are now focusing on determining whether there is a direct correlation between being more intentional about asking these questions and declining patient infection and safety event rates.

Patient satisfaction scores improved last year but have since plateaued. Baseline data from November 2014 showed a score of 75.0 and in June 2016 it was 80.5. Focused work is happening to engage the team and further improve the results.

Based on three cycles of the internal survey, data shows the percentage of staff who knows what is expected of them on rounds improved from $62 \%$ to $82 \%$ overall with 3 of the 6 roles now at $100 \%$ understanding (see figure \#1).

Resident teaching scores are now reviewed quarterly instead of semi-annually. Comparing November 2014 with June 2016, teaching scores have shown a statistically 
Percentage of Respondents Answering 4 or 5

"I Know What's Expected of Me in Rounds"

(Likert Scale)

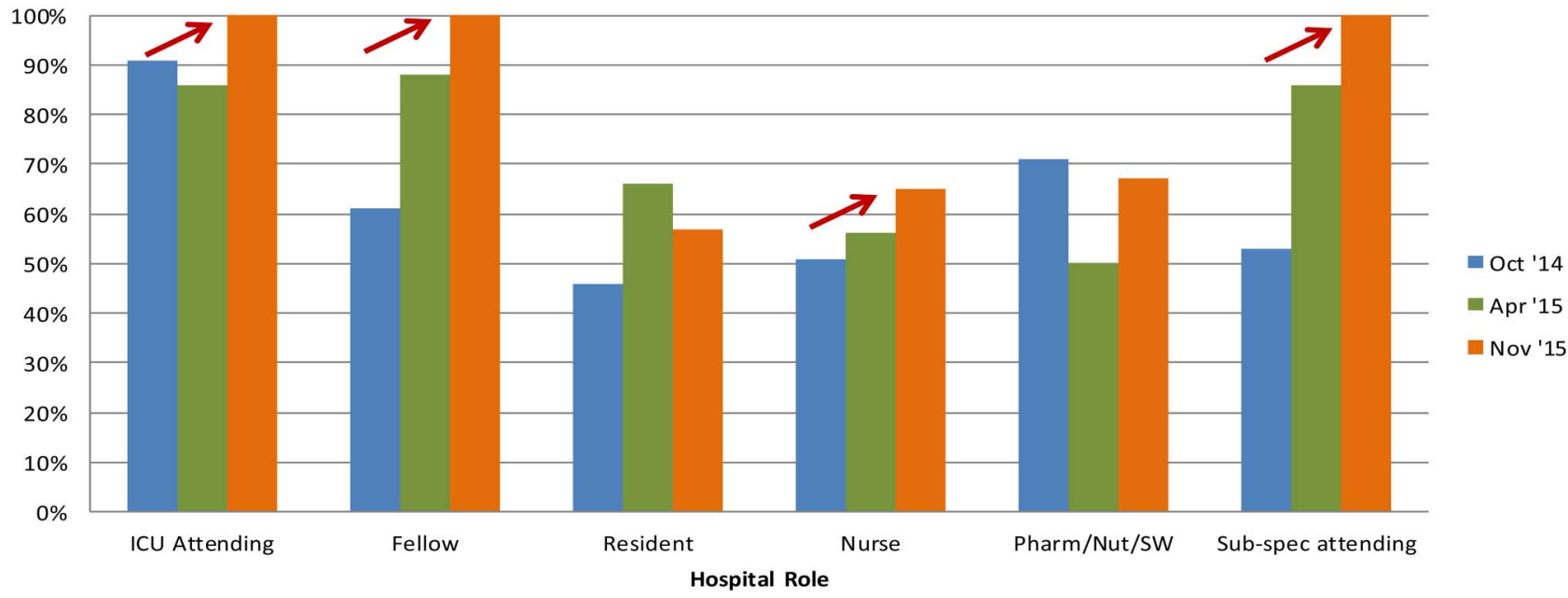

Figure 1

significant increase of about 0.3 on a Likert scale (see figure \#2) (See supplementary file - PICU Rounding Improvements Results Slides).

\section{LESSONS AND LIMITATIONS}

We have often reflected on the success with this project. We feel that it is because we engaged a team of thinkers and do-ers and, thus, we had both vision and progress. We had regular meetings, shared accountability and comradery between physician and nursing partners. We had a collective agreement on the current problem and the overall purpose of the improvement work. We had support from the top, a willingness to try new things and a commitment to better serve our patients. Most importantly, we had physician and nursing leaders out doing the work and being part of the solution to manage change. We surmised that a lack of multidisciplinary engagement in the prior PICU rounding improvement projects was an important factor in lack of sustainability of those efforts.

While several observations were completed before beginning the project, the core baseline of four hour rounds in the PICU was somewhat anecdotal based on the experiences of the attendings over the past couple of years. Direct feedback included complaints and 
frustration from sub-specialists about not knowing the time rounds would occur for their specific patients. Primary care team members also lamented that they were not able to complete their daily work in a timely manner due to the duration of rounds pushing work into the afternoon. The most important piece of feedback received from PICU care team members was that the patient care was not being advanced throughout the day, which led to delays in care and potentially to longer stays on the critical care unit. Additional insight from PICU attendings was that, because of the burdens within the work day, they were not able to complete their required documentation until very late into the evening, that personal work-life balance suffered and burn-out was occurring.

One of the principles of this work was to not create extra work for already busy faculty. Thus, it was necessary to incorporate data collection into the attending daily work with a simple method of real-time self-auditing instead of external observers performing direct observation. It is possible that the auditing process in and of itself may have introduced bias and caused providers to shorten rounds. Whether improvement was directly a result of the structural changes or an indirect affect of measurement, both would have a positive affect on the overall goal of increasing rounding efficiency.

Shortening rounds released other team members to continue working on other duties not related to direct patient care (i.e. total parental nutrition validation, evaluate sedation tapers, precept new staff/students, or attend committee meetings). This resulted in significant indirect cost savings to the organization by creating additional capacity within each role.

Variation: when census was low, sometimes per patient average rounding times increased perhaps due to the luxury of having extra time to offer additional teaching and discussion.

This project is highly generalized and can be replicated. For example, efforts are underway to spread the methodology to the Cardiac Intensive Care Unit (CICU) within the medical center and data collection has begun with their new rounding sheet. Other internal departments and external institutions have reached out for the tools, templates, and replication guide for this work. Hospitalists and General Surgeons have requested use of the Quality \& Safety checklist that has also been recognized as best practice by senior administrators.

Limitations: This team's improvement efforts were designed in a teaching hospital. Results in other settings may vary. An additional potential limiting factor is the feasibility of having a project manager to develop and analyze data for measurement and to maintain forward momentum of the project.

\section{CONCLUSION}

The team began with a problem that rounds in the PICU at Seattle Children's Hospital were too long, too variable in content, and did not reliably meet the needs of the patient. Through various interventions, described in this paper, rounding times were reduced from four hours/day to two hours/day and engagement was improved for all roles on the care team. Standard work now exists in many phases of rounds where it was not present before. While preparing for this project, the team knew that changing the culture would be a significant part of the task at hand. The medical leadership engaged faculty colleagues from the beginning to articulate current state and share the vision for a new ideal state. Consistent check-in meetings helped keep up the momentum of the improvements. Currently, data for rounding duration and average time per patient is being collected consistently. Quality \& Safety indicators were improved again recently as an online tool has been created within the electronic medical record. Our work continues to more effectively meet the needs of the families, and improvement has been sustained with consistent communication between faculty and nursing. By comparison, improvements to decrease rounding time and increase employee satisfaction are similar to the reference that was cited.

Acknowledgements Other improvement team members: Michael Portman, Mark Reed, Chris Parrott as well as all faculty and staff in the Seattle Children's Hospital Pediatric Intensive Care Unit.

Declaration of interests Nothing to declare.

Ethical approval The Seattle Children's Hospital Institutional Review Board (IRB) determined that this quality improvement activity is not research involving human subjects, and therefore IRB review and approval is not required.

Open Access This is an open-access article distributed under the terms of the Creative Commons Attribution Non-commercial License, which permits use, distribution, and reproduction in any medium, provided the original work is properly cited, the use is non commercial and is otherwise in compliance with the license. See:

- http://creativecommons.org/licenses/by-nc/2.0/

- http://creativecommons.org/licenses/by-nc/2.0/legalcode

\section{REFERENCE}

1. Vats A, Goin KH, Villarreal MC, Yilmaz T, Fortenberry JD, Keskinocak $P$. The impact of a lean rounding process in a pediatric intensive care unit. Crit Care Med. 2012 Feb;40:608-17 